\title{
OVARIAN HAEMANGIOMA WITH INFUNDIBULOPELVIC LIGAMENT TORSION IN A 13-YEAR-OLD GIRL
}

\author{
Zganjer V. ${ }^{1}$, Zganjer M. ${ }^{2}$, Cigit I. ${ }^{2}$, Tomicic H. ${ }^{3}$, Cizmic L. ${ }^{4}$ \\ ${ }^{1}$ Pfizer d.o.o. Radnicka 80, 10000 Zagreb, Croatia \\ ${ }^{2}$ University Hospital „Sestre Milosrdnice“, Department of Pediatric Surgery, Klaićeva 16, 10000 Zagreb, \\ Croatia \\ ${ }^{3}$ University Hospital „Sestre Milosrdnice“, Department of Surgery, Draškovićeva 19, 10000 Zagreb, \\ Croatia \\ ${ }^{4}$ Faculty of Medicine, University of Zagreb, Croatia
}

\begin{abstract}
Background. Ovarian haemangioma are very rare type of tumors, although the ovary itself is a highly vascularised organ. Such benign tumors of the blood vessels are rare in ovaries during childhood.

Clinical case. We described a 13-year-old girl with an ovarian haemangioma presented clinically with acute abdomen symptoms - abdominal and pelvic pain and vomiting. The pelvic mass was noted and sonography revealed a huge cystic tumor with probably ovarian torsion. A left salpingo-oophorectomy and appendectomy were performed with an uneventful postoperative course. Pathology examination revealed a cavernous haemangioma of the ovary.

Conclusion. Haemangiomas of the ovary, especially in children, are very rare type of the benign tumor. Surgical extirpation of the involved areas is the treatment of the choice.
\end{abstract}

Key words: haemangioma, ovary, ovarian torsion, children

\section{INTRODUCTION}

Infantile haemangiomas are described as a benign (non-cancerous) condition affecting cutaneous blood vessels. It is also known as proliferative haemangioma because it is due to proliferating endothelial cells. Haemangiomas usually develop shortly after birth and they are distinct from vascular malformations, which are usually present at birth and are less common. Over $80 \%$ of infantile haemangiomas occur on the head and neck area. They grow to $80 \%$ of maximum size in the first three months and most stop growing at about 5 months. However, they may keep growing for up to 18 months $(1,2)$. Although ovaries have a very rich vascular blood supply, haemangiomas of the ovary are extremely rare especially in ovaries during childhood. The number of welldocumented cases of ovarian haemangioma, in both adult and children, does not exceed 100 patients.

Haemangioma of the ovary was first described by Payne in 1869 (cited by Talerman) $(3,4)$. Mostly they are the small tumors discovered incidentally. In low percentage of cases ovarian haemangiomas presented clinically with an acute abdomen. The presenting symptoms of acute abdomen and the ultrasonographic study in most cases led to the preoperative diagnosis of torsion of an ovarian tumor. When we found a tumor on the ovaries, in the most cases ovarian cysts are present. Ovarian cysts are most commonly in children undergoing the conservative treatment, but larger cyst or ovarian torsion need surgical treatment. The purpose of this presentation is how to deal with changes in the ovaries. Only cysts of smaller dimensions could be treated conservatively. Ovarian cysts larger than $5 \mathrm{~cm}$ and tumors should be treated surgically due to the possibility of malignant alteration and / or ovarian torsion (5).

Address for correspondence:

Mirko Zganjer, MSc.PhD,University Hospital „Sestre Milosrdnice“, Department of Pediatric Surgery, Klaićeva 16 , 10000 Zagreb, Croatia

Phone: +385 914600 143; Fax: +385 14600 169; e-mail: mirko.ganjer@gmail.com 


\section{CASE REPORT}

A 13-years-old girl was admitted to the surgical clinic with severe pain in the abdomen which lasted for 24 hours. Prior hospitalization she was vomiting 6 times. After being admitted to the hospital, we started with the treatment. Inflammatory parameters were elevated, Sedimentation rate was $65 \mathrm{~mm} / \mathrm{h}$ ( normal 0-15 mm/h), leukocyte count was 18.9 (normal 4-12x109/L) and C-reactive protein was 82mg/L (normal<5mg/L). Patient was immediately subjected to abdominal ultrasound (Figure 1). In ultrasonographic evaluation of our patient a pelvic mass was found located at the left ovary. Ovary size was $10 \times 6.4 \mathrm{~cm}$, which had no blood flow. Ultrasound showed a large ovarian torsion. Then she was examined by a gynaecologist. Rectal and abdominal ultrasound transducer probe found solid pelvic mass with impaired flow through it. We suspected ovarian torsion and immediately surgery was induced. We have not even attempted laparoscopic operative treatment. The pain lasted a long time, an ultrasound showed torsion of the ovary and we were not sure they could perform surgery with laparoscopic management of the mass and untwisting the ovary and the tube. A Pfannenstiel incision was done and displayed left ovarian tumor the size of $10 \times 7 \times 4 \mathrm{~cm}$. Ovary with the tumor and the fallopian tube was turned 5 x 180 degrees. Fallopian tube and ovary with the tumor were necrotic and the only treatment option was the removal of the tumor, ovaries and fallopian tubes (Figure 2). The right ovary was adequate layout and size for gestational age girls and grossly was unremarkable. During surgery we made removal of the appendix. We always removed appendix when surgery in the abdomen is necessary for other reasons. It is known that after abdominal surgery adhesions are present, with appendectomy we prevent re-surgery which may require an appendicitis. Postoperative course was uneventful, the wound healed properly. Tumor markers including CA125 ( cancer antigen 125), and CEA (carcinoembryonic antigen) were within normal limits / normal values: CA125 < 35 mU/L; CEA $<3.4 \mathrm{~g} / \mathrm{L} /$.

Removed parts were sent for histopathological diagnosis. Fallopian tube was $5 \mathrm{~cm}$ long filled with blood. In addition to the Fallopian tube was an oval lesion size

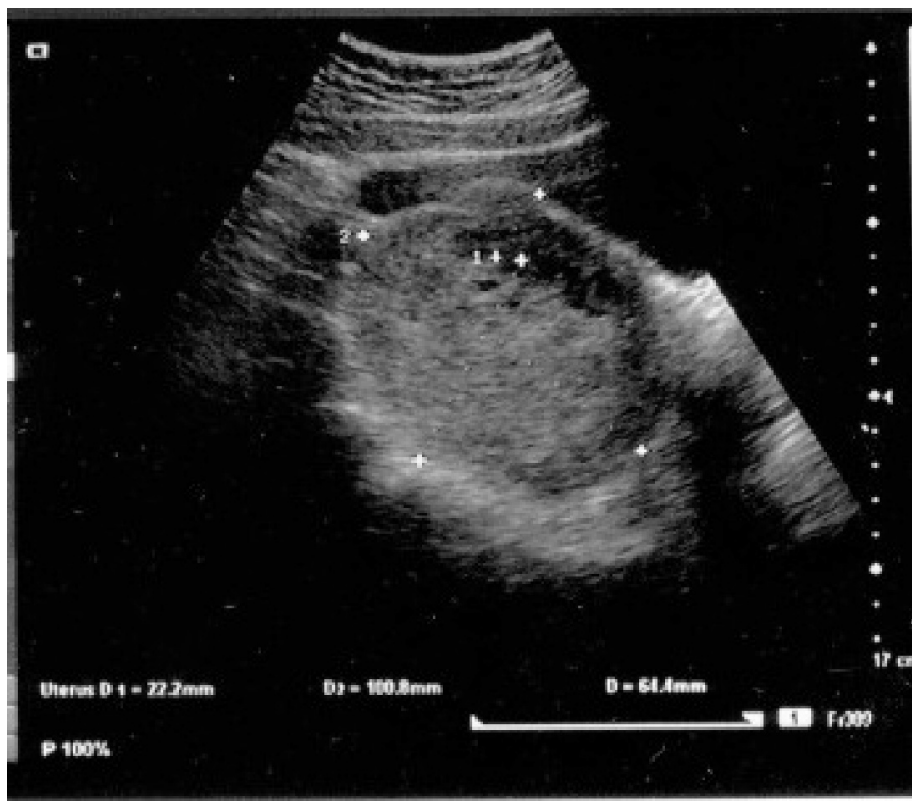

Fig. 1. Abdominal ultrasound with ovarian torsion 


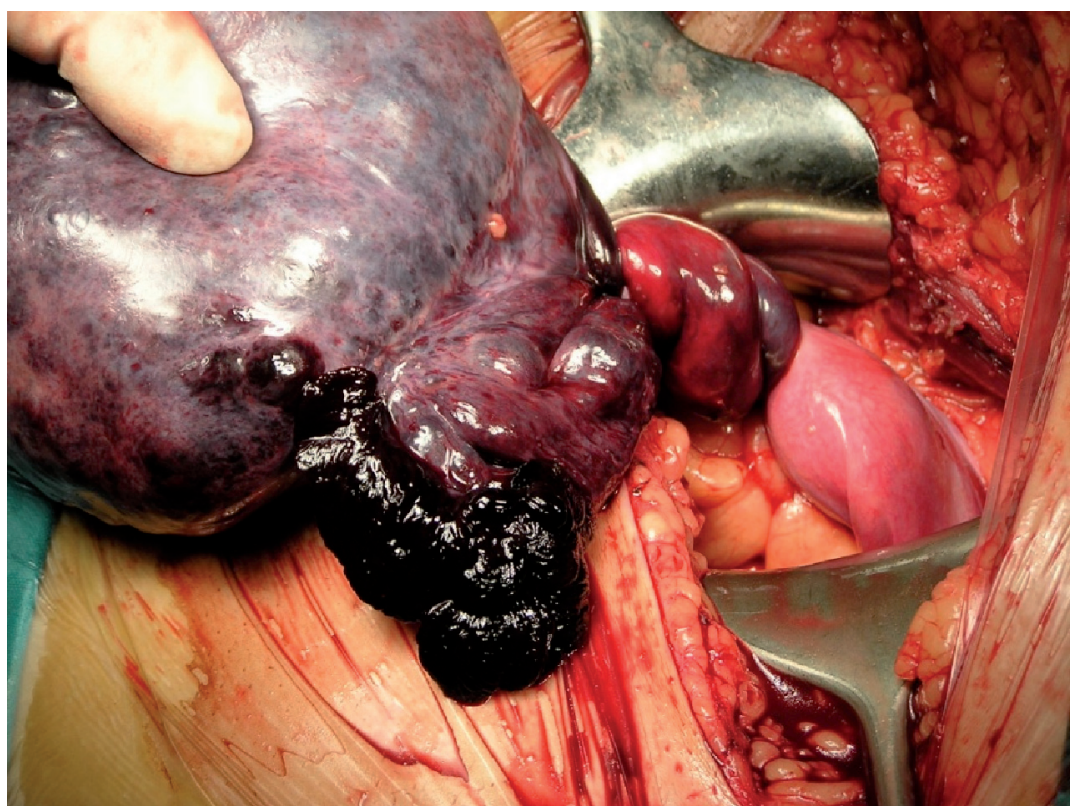

Fig. 2. Haemangioma of the ovary with torsion and necrosis

$10 \times 7 \times 4 \mathrm{~cm}$. The formation of the cut was fully encapsulated and haemorrhagic. Macroscopically, the tissue of the ovary was not found. Microscopically examinations was made of 23 slices and find different wide vascular spaces, in some places, spaces were dilated with a thick or thin-walled space. Described properties were lined with flattened endothelium and filled with erythrocytes. Histologically, there were parts as ovarian and fallopian tube and tumor formation are fully imbued with bleeding, ovarian structure was lost to necrosis. Histological picture, histochemistry (Mallory) and immunohistochemical (CD31, D2-40) analysis of the received and reviewed materials corresponds to haemangioma of the ovary with torsion of the ovary.

\section{DISCUSSION}

Haemangiomas are benign lesions arising from a failure in vascular formation forming abnormal vascular channels and are of two types- capillary and cavernous. The blood vessels in uppermost layers of the skin are dilated and called capillary haemangioma. Deep infantile haemangiomas are also called cavernous haemangiomas and they appear as a bluish soft to firm swelling. The difference between them relates to the size of the blood vessels formed. They may be localised (confined to a small area) or segmental (involving a larger neuroectodermal unit). The superficial infantile haemangioma is also called capillary haemangioma, capillary naevus, strawberry haemangioma, strawberry naevus, and haemangioma simplex. Capillary haemangiomas are made of the blood vessels of the caliber of normal capillaries, and cavernous types consist of larger channels which are more deeply set in the dermis and subcutis. Both types of haemangioma may occur together in mixed angiomatous naevi $(1,5)$. Vascular tumors of the female genital tract, especially those arising in the ovary in childhood, are very rare although the ovary has rich blood supply (6). The most cases of the ovarian haemangioma are small and incidental findings at operation. Large lesion tends to present clinically with symptoms typical for presence of the mass or such as abdomi- 
nal pain due to torsion. In our case haemangioma was large and symptomatic such as acute abdomen due to torsion (7-9). Cavernous haemangioma of the ovary is more prevalent than other histological types. The etiology of the ovarian haemangioma is unknown. Some authors think that the hemangiomas are hamartomas, true tumors, stimulated the blood vessels or congenital malformations $(10,11)$.

Our surgical approach is debatable because some would strongly advocate for laparoscopic management of the mass, and untwisting the ovary and tube; the literature seems to support this approach. In tumors changes in the ovary, where there is no torsion or torsion of the ovary takes up to 6 hours we always do laparoscopic surgery. In ovarian torsion lasting as long in our patient, always choose the classic surgery. In torsion of the ovary that last longer than 24 hours, the success of laparoscopic surgery was low in our hospital. On several occasions we tried to do laparoscopic untwisting the ovary and tube and in most cases the surgery could not be done for strong edema. A Pfannenstiel incision is often used in preference to other incision types for the sake of aesthetics, because the scar will be hidden by the pubic hair. The incision does not distort the belly button and heals faster than the traditional vertical incision. A Pfannenstiel incision is a type of surgical incision that allows access to the abdomen. Because the exposure is limited this incision should be used only when surgery is planned on the pelvic organs. The Pfannenstiel incision offers large view of central pelvis but limits exposure to the lateral pelvis and upper abdomen, factors that limit the usefulness of this incision for gynecologic cancer surgery.

Haemangioma of the ovary, especially in children, is very rare neoplasm. Surgical extirpation, laparoscopic or open method, of the involved areas is the treatment of the choice. After histopatological examination and correct diagnosis, definitive surgical treatment was done. In our patient we had only one method of treatment because it was haemangioma with an ovarian torsion and both of them were necrotic.

\section{REFERENCES}

1. Holland KE, Drolet BA.Infantile hemangioma. Pediatr Clin North Am. 2010;57(5):1069-83.

2. Mendiratta V, Jabeen M.Infantile hemangioma: an update. Indian J Dermatol Venereol Leprol. 2010;76(5):469-75.

3. Payne JF: Vascular tumors of the liver, suprarenal capsules and other organs. Trans Path Soc London 1869; 20: 203.

4. Talerman A. Hemangiomas of the ovary and the uterine cervix. Obstet Gynecol 1967; 30: $108-113$.

5. Haggstrom AN, Drolet BA, Baselga E, et al. „Prospective study of infantile hemangiomas: clinical characteristics predicting complications and treatment“. Pediatrics.2006;118 (3): 882-7.

6. Gupta R., Singh S., Nigam S., Khurana N.Benign vascular tumors of female genital tract. International Journal of Gynecological Cancer. 2006.16(3);1195-1299.

7. M'pemba Loufoua-Lemay AB, Peko JF, Mbongo JA, Mokoko JC, Nzingoula S. Ovarian torsion revealing an ovarian cavernous hemangioma in a child. Arch Pediatr. 2003;10(11):986-8.

8. Correa-Rivas MS, Colón-González G, Lugo-Vicente H.Cavernous hemangioma presenting as a right adnexal mass in a child. P R Health Sci J. 2003;22(3):311-3.

9. Kloss BT, Prince LA. Ovarian torsion in a prepubertal girl. Int J Emerg Med. 2009 Mar 24; 2(2):127-8.

10. Uppal S., Heller DS., Majmudar B. Ovarian hemangioma-report of three cases and review of the literature. Arch Gynecol Obstet.2004; 270:1-5.

11. Mirilas P, Georgiou G, Zevgolis G.Ovarian cavernous hemangioma in an 8-year-old girl. Eur J Pediatr Surg. 1999;9(2):116-8. 